\title{
El estilo de gobernanza en la cadena de valor de la industria del acero en México
}

\author{
Manuel Alexis Vázquez Zacarías* \\ Mónica Blanco Jiménez \\ Jesús Cruz Álvarez
}

\begin{abstract}
Resumen
La producción de acero es uno de los indicadores vinculados al crecimiento económico de los países, al ser utilizado éste como materia prima de diversas industrias. Razón por la cual en este estudio se presenta la importancia de la producción del acero en México, su cadena de valor y el tipo de relación que existe entre los clientes y proveedores. Por lo tanto, el objetivo es identificar el estilo de gobernanzaque seaplica en la cadena de valor de la industria del acero en México, enfatizando el rol de la empresa líder. La metodología utilizada es cualitativa de tipo descriptiva; con un análisis teórico de la literatura se determinó que las empresas que forman parte de la cadena de valor del acero en México se ven beneficiadas de tener una gobernanza de tipo cautiva, debido a que la empresa líder las ayuda ensu crecimiento y mejoras de procesos.
\end{abstract}

\begin{abstract}
The steel production is one of the indicators related to the economic growth of countries, to be used as raw material for various industries. This study shows the importance of steel production in Mexico, its value chain and the type of relationship between customers and suppliers. Therefore, the objective is to identify the style of governance that applies in the value chain of the steel industry in Mexico, emphasizing the role of the leader. The methodology used is qualitative descriptive; with theoretical analysis of the literature found that the companies thatare part of the value chain of steel in Mexico benefit of having a captive governance are kind because the leader aid in growth and processimprovements.
\end{abstract}

Palabras clave: industria del acero, cadena de valor, gobernanza, México.

Keywords: steel industry, value chain, governance, Mexico.

Universidad Autónoma de Nuevo León, facpya, Ciudad Universitaria, San Nicolás de los Garza, tel : 81-83294080 Correo electrónico: manuel vazquezzcr@uanl edu mx 


\section{Introducción}

La cadena de valor es un eslabonamiento de actividades que muestran desde la producción hasta la entrega final del producto al consumidor, considerando las diversas actividades intermedias de producción que necesitan ser vinculadas (Kaplinsky y Morris, 2009) Una muestra de este tipo de eslabonamientos es la cadena del acero, que se caracteriza por una larga serie de fases productivas que comprende desde la industria básica, fases de procesamiento industrial, hasta la comercialización de productos, los cuales podrán ser incorporados dentro de otras cadenas de valor orientadas a un segmento específico (Chiviri y García, 2011).

Esta relación de encadenamiento impacta de forma directa a los clientes y proveedores, debido a que cuando una empresa solicita ciertos requerimientos a sus proveedores, entonces se genera una relación que involucra coordinación y poder. Los investigadores Gereffi, Humphrey y Sturgeon (2005) señalan que los criterios de coordinación y poder permiten entender las distintas formas en las que se gobiernan los esfuerzos de eslabonamiento de las empresas en las cadenas de valor

Dada la naturaleza de este eslabonamiento, en esta investigación se realiza un estudio de caso que pretende mostrar la situación de la estructura de la cadena de valor de la industria del acero en México y su estilo de gobernanza Particularmente la pregunta de investigación es: ¿Qué tipo de gobernanza se ejerce en la cadena de valor del proceso productivo del acero en México? Por lo que el objetivo principal es identificar el estilo de gobernanza que se aplica en la cadena de valor de la industria del acero en México, haciendo hincapié en el diagrama de la cadena de valor y en los principales atributos, lo que permite entender la relación proveedor-cliente que tienen estas empresas dentro de la cadena productiva de esta industria y que la empresa líder pueda aplicar programas que permitan mejorar esta relación

En la primera sección de este artículo se presentará el panorama de la industria del acero en México y las características y atributos de su cadena de valor En la segunda parte se presenta el marco teórico que le otorga fundamento a los criterios de gobernanza No se pretende analizar con detalle las características de las principales empresas productoras ni de sus proveedores; se parte de un análisis estadístico que presenta las principales empresas en 
México y cómo se realiza la estructura de la cadena de valor en esta industria En la tercera sección de este artículo se expondrá la metodología descriptivacualitativa que se utilizó, y en la cuarta parte se identificará como resultado el tipo de estilo de gobernanza que se aplica en la relación clientes-proveedores de la cadena de valor de la industria del acero

\section{Panorama de la industria del acero en México y su cadena de valor}

Presentando la evolución de la producción del acero en México, podemos observar en la gráfica 1 que presenta un crecimiento considerable de $37 \%$ en su producción en los últimos 15 años, pasando de 13 millones de toneladas en 1996 a 181 millones en 2013 según datos de la Cámara Nacional de la Industria del Hierro y del Acero en México (Canacero, 2013) Cabe destacar que esta producción mexicana representó $12 \%$ del total de la producción mundial al año 2011 y México se convirtió en 2014 en el treceavo productor de acero en el mundo (Canacero, 2015)

\section{Gráfica 1}

Producción de acero en México de 1996 a 2013

(Millones de toneladas)

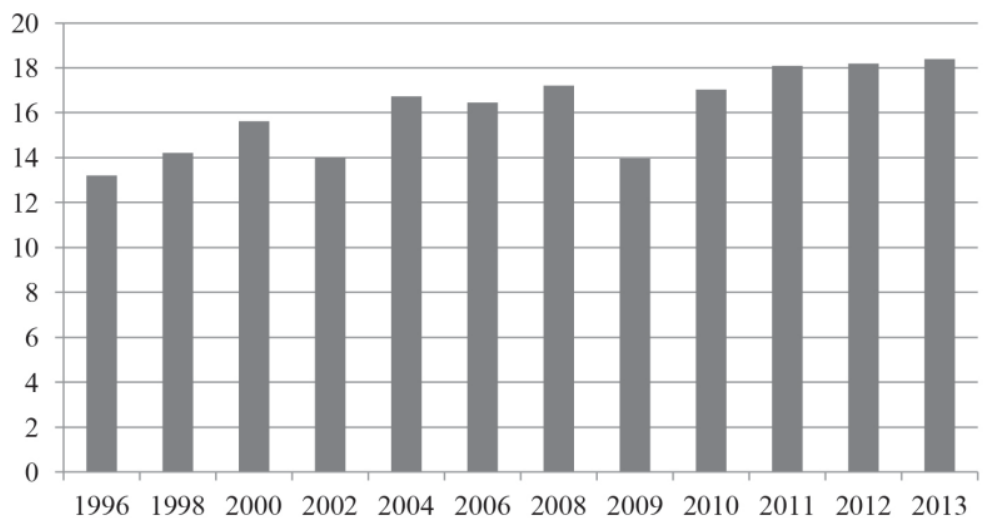

Fuente: Canacero (2015) a partir de www canacero org

Acorde con la Canacero (2013), en México la cadena de valor del acero se ramifica y deriva en seis principales aplicaciones para la generación de 
productos terminados en las siguientes industrias: laindustria metalmecánica básica, la fabricación de productos metálicos, la fabricación de maquinaria y equipo, la fabricación de equipos de cómputo y accesorios eléctricos, la fabricación de aparatos eléctricos y la fabricación de equipo de transformación Esta sectorización permite una distribución de la utilización del acero en México (gráfica 2) que está orientada principalmente a la industria de la construcción con $512 \%$, seguida por maquinaria metálica con $1450 \%$ y con $12 \%$ para los productos metálicos y la industria automotriz

\section{Gráfica 2}

Porcentajes de utilización del acero en México

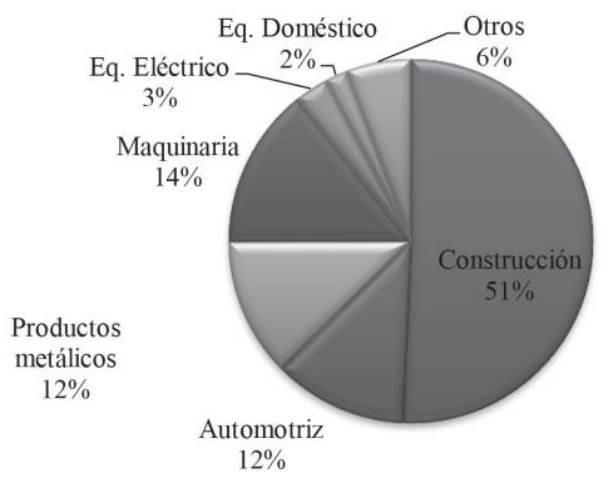

Fuente: elaboración propia con base en iris (2013)

En cuanto a la distribución geográfica de la industria del acero en México, la Canacero (2013) establece que la mayoría de las plantas de producción y centros de distribución se concentran en las regiones noreste y centro del país, como se observa en la figura 1, que incluyen los estados de Coahuila, Nuevo León, Tamaulipas, San Luis Potosí, Jalisco, Guanajuato, Michoacán, Estado de México, Tlaxcala, Puebla y Veracruz; también existe actividad en los estados de Baja California y Yucatán De esta manera se puede señalar que $406 \%$ del territorio mexicano está vinculado de manera directa con la industriadelacero 
Figura 1

Distribución geográfica de las principales empresas del acero

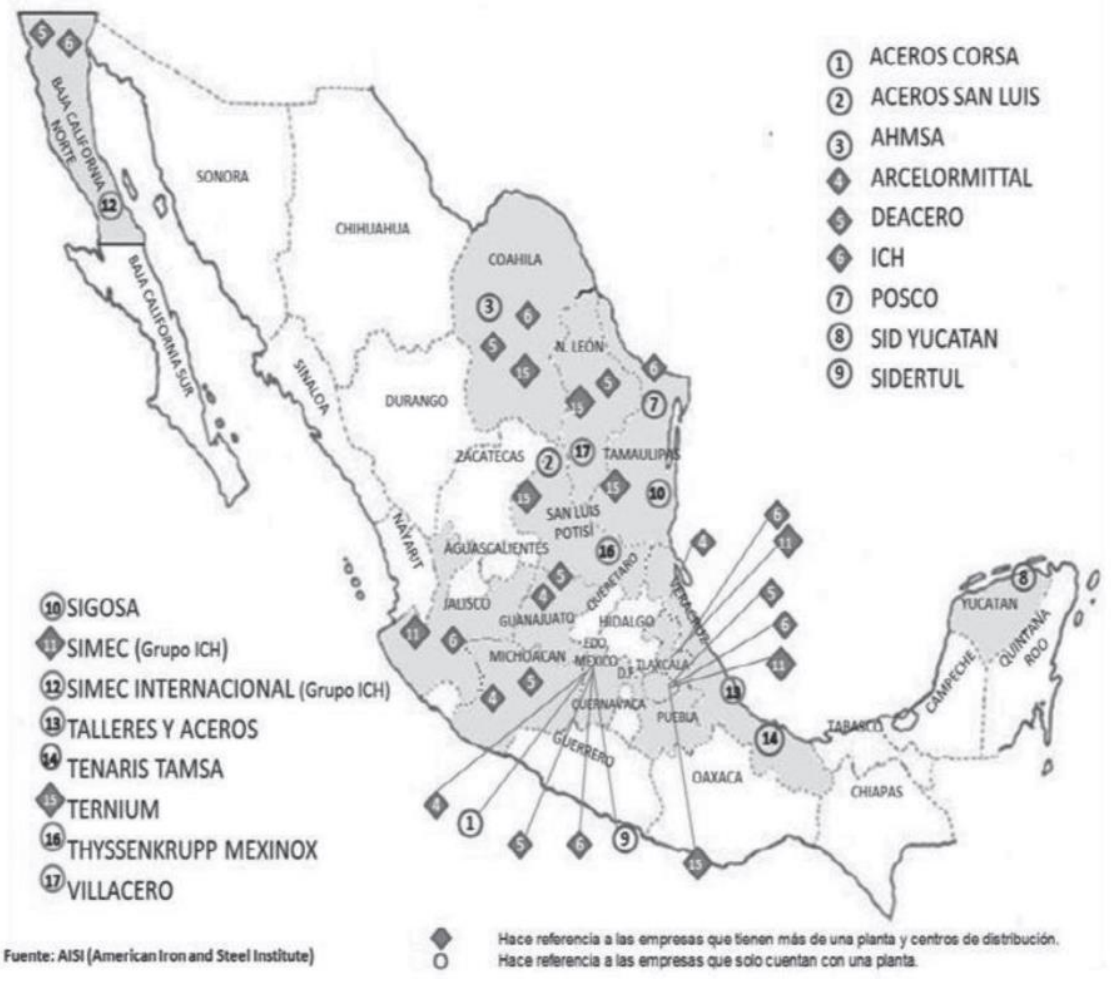

Fuente: Canacero, 2013

En cuanto a las empresas, en México las principales cinco productoras de acero son Arcelormittal, ahmsa, Ternium México, DeAcero y tamsa, que en conjunto concentran $85 \%$ de la producción nacional de acero El $15 \%$ restante de la producción nacional de acero lo realizan otras acerías como ich, Grupo San Luis, Aceros Corsa y Grupo simec, entre otras (Canacero, 2013)

Después de la presentación breve del panorama general de la industria del acero en México respecto a su crecimiento y las principales empresas productoras, se requiere mostrar la forma en la que están amalgamadas las actividades principales realizadas por esta industria Particularmente, es en este punto donde la cadena de valor tiene gran importancia, debido a que la cadena es una descripción de las actividades necesarias que muestran desde 
la concepción hasta el producto final para al consumidor, considerando las diversas actividades intermedias de producción que necesitan ser vinculadas (Kaplinsky y Morris, 2009)

De acuerdo con Chiviri y García (2011), la cadena del acero se caracteriza por una larga serie de fases productivas que comprenden desde la industria básica, fases de procesamiento industrial, hasta la comercialización de productos, los cuales podrán ser incorporados dentro de otras industrias como lo es la del sector metalmecánico

Realizando un análisis con detalle, en la primera parte de la cadena del acero se pueden identificar tres eslabones principales: materias primas, productos intermedios y productos terminados, que se presentan en la figura 2 Asimismo, a partir de los procesos de fabricación que involucran horno eléctrico o altos hornos es posible identificar que se generan tres productos intermedios, que son el planchón, palanquilla y tocho Por último es vital resaltar que utilizando los productos intermedios se pueden derivar los productos finales, que se mencionan a continuación:

- Los derivados principales del producto-intermedio de planchón son los laminados delgados, que son usados principalmente para enseres domésticos; los laminados gruesos tienen aplicaciones industriales, mientras que los rollos tienen aplicaciones diversas para su transformación por medio de procesos metalmecánicos diversos

- Los productos derivados de la palanquilla, los cuales son usados principalmente para la construcción, entre los cuales destacan las varillas para armados, tuberías diversas y variedades de calibres acerados

- Los productos derivados del tocho son usados para la fabricación de perfiles estructurales de aplicaciones en la construcción, forjas y maquinados de grueso calibre

\section{Desarrollo de la teoría sobre los tipos y criterios de gobernanza}

Para entender la forma en la que se gobiernan los esfuerzos de las empresas de eslabonamientos mediante coordinación y poder, los investigadores Gereffi, Humphrey y Sturgeon (2005) desarrollaron los siguientes criterios de la actividad que se llama gobernanza De acuerdo con estos autores es 
Figura 2

Estructura de la cadena de valor del mercado del acero en México

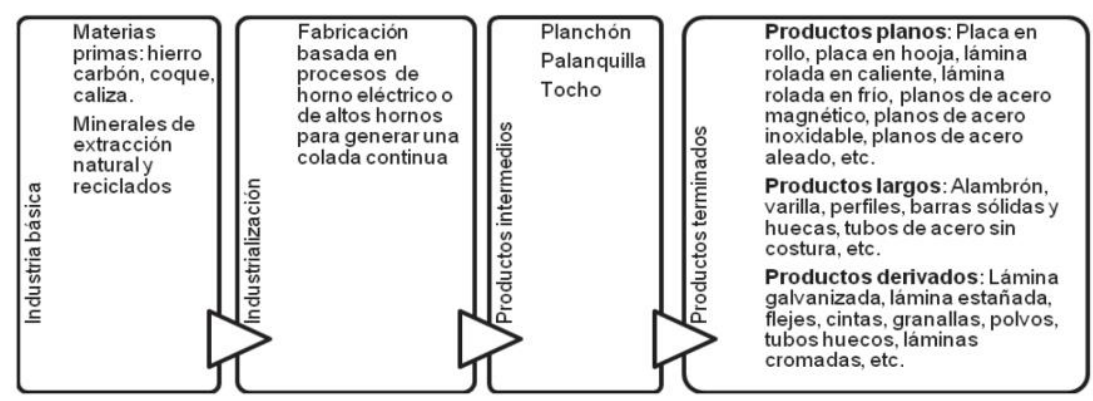

Fuente: elaboración propia con base en Camacho, 2011

posible identificar en las cadenas de valor cinco diferentes formas de gobernanza:

1 Gobernanza de mercado. Se da cuando las relaciones entre las empresas están basadas en el precio y los costos, por lo que para cambiar de relación con otro proveedor se basan en los costos bajos, por lo que existe una muy baja asimetría de poder, ya que tanto el proveedor como el cliente trabajan con múltiples socios

2 Gobernanza modular. Se encuentra en relaciones donde el proveedor produce de acuerdo con las especificaciones del cliente. Se debe considerar que en estos casos el proveedor emplea maquinaria genérica, lo que implica que no requiere una inversión en tecnología muy detallada para satisfacer los criterios del cliente, por lo que existe una baja asimetría de poder ya que se puede trabajar con múltiples socios

3 Gobernanza relacional. Se encuentra en relaciones hechas con interacciones complejas debido a que existe un poder equilibrado o simétrico, basado en una dependencia mutua entre el proveedor y el cliente Incluso se tienen activos muy específicos que se utilizan para una sola relación.

4 Gobernanza cautiva. Se da cuando el proveedor de tamaño pequeño tiene como cliente una empresa grande que está constantemente monitoreando y controlando al pequeño proveedor Se puede decir que la empresa grande tiene mayor poder respecto al proveedor pequeño 
5 Gobernanza de jerarquía. Se caracteriza por el hecho de que la empresa líder está verticalmente integrada Aquí se tiene el poder más dominante debido a que la administración tiene un control que fluye a los subordinados.

Para identificar cuál de las cinco formas de gobernanza es la que tiene una cadena en particular, se torna necesario considerar tres criterios que según Gereffi y sus colegas (2005) se pueden evaluar como bajo o alto, según los siguientes parámetros:

- El primer criterio es la complejidad de la información transferida respecto a las especificaciones del producto y proceso.

- El segundo es el grado en que la información puede ser codificada y transmitida eficientemente.

- El tercero son las capacidades que tienen los proveedores en relación con los requerimientos de la transacción

\section{Análisis metodológico}

Es una investigación descriptiva que pretende presentar la evolución de la industria en México y la estructura de la cadena de valor, para después describir con el estudio de caso cualitativo el tipo de gobernanza que se adopta en esta industria

El método de estudio de caso cualitativo sigue un enfoque que facilita la exploración de los fenómenos dentro de su contexto usando una variedad de fuentes de datos (Baxter y Jack, 2008) Particularmente, este método va acorde con lo que se pretende hacer en esta investigación, ya que mediante una revisión de múltiples fuentes secundarias se realizará una descripción de cómo es la cadena de valor del acero en el contex to de México

Yin (2003) afirmó que un diseño de estudio de caso se debe utilizar si dos características están presentes La primera es que el comportamiento de los participantes en el estudio no pueda ser manipulado, y esto está presente en la investigación realizada aquí, debido a que las actividades de la cadena son realizadas por empresas independientes La segunda característica es que las condiciones contextuales deben ser consideradas Esta última característica también está presente en este estudio debido a que las actividades involucradas en la cadena de valor se derivan solamente del contexto mexicano 
De acuerdo con Yin (2003), una vez tomada la decisión de la utilización del estudio de caso como metodología de investigación, ahora el caso debe ser clasificado como explicativo (muestra vínculos causales que son demasiado complejos para una encuesta), exploratorios (existen resultados no claros de laintervención analizada), o descriptivo En este estudio, el caso es del tipo descriptivo debido a que se intentará establecer la cadena de valor del acero en México enfatizando su tipo de gobernanza

Los pasos que se siguieron para el desarrollo de la cadena de valor del acero en México consistieron primero en identificar los principales procesos, comenzando por las materias primas e insumos utilizados en dichos procesos, para producir productos intermedios y qué tipo de productos se derivan de éstos. Finalmente se identifican los tipos de gobernanza y los criterios para clasificarlos, permitiendo así determinar cuál es la que se aplica a este estudio de caso Los pasos descritos previamente se llevaron a cabo a través de una extensa revisión de fuentes secundarias, como son los artículos de investigación, los materiales publicados en la Canacero y la información publicada por la Secretaría de Economía de México y varias entrevistas con los responsables de la empresalíder

\section{Resultados}

En la sección de revisión de literatura se mencionó que existen cinco formas de gobernanza para las cadenas de valor y que para poder identificar cuál se tiene es necesario evaluar (como bajo o alto) los criterios de complejidad, codificación de la información y capacidad del proveedor. Por lo tanto, se realizó un análisis de los tres criterios previamente descritos en la sección de revisión de literatura y se aplicaron a la cadena de valor de la industria del acero en México con base en los estudios de los informes de actividad de la industria del acero presentadas por la Canacero y de entrevistas a asesores de la industria del acero en Nuevo León (véanse cuadros 1, 2 y 3 ) 


\section{Cuadro 1 \\ Criterios de gobernanza de la cadena de valor delaceroen México segúnel criterio 1}

\begin{tabular}{|c|c|c|}
\hline Criterio & Presencia en la cadena & Eva. \\
\hline $\begin{array}{l}\text { Complejidad } \\
\text { de la } \\
\text { Información }\end{array}$ & $\begin{array}{l}\text { Actualmente existen en el mercado una gran variedad de usos } \\
\text { para el acero como producto intermedio en diferentes industrias } \\
\text { (construcción, naval, mecánica, petrolera, etc.). Particularmente, } \\
\text { lacomplejidad alta en elacero se puede identificaren elhecho } \\
\text { dequealserunproducto queseusa como insumoen múltiples } \\
\text { industrias, entonces esto ocasiona que las características del } \\
\text { acero estén evolucionando para cumplir con las necesidades } \\
\text { puntuales de cada uno de los distintos clientes. Incluso, otra } \\
\text { forma de percatarse de la complejidad alta de la información } \\
\text { en el acero es mediante los avances tecnológicos acelerados } \\
\text { en los diversos campos de la ingeniería en los que se usa el } \\
\text { acero con unas características que sí se pueden aplicar en una } \\
\text { industria cliente pero difícilmente se trasladan a otra industria } \\
\text { cliente (Altos Hornos de México, 2013). Asimismo, debido a la } \\
\text { complejidad es que constantemente se hacen esfuerzos para } \\
\text { categorizar los constantes avances tecnológicos y poder esti- } \\
\text { pular especificaciones para el acero que puedan establecerse } \\
\text { como requerimientos para los proveedores y/o características } \\
\text { del producto que estará recibiendo el cliente de acuerdo con sus } \\
\text { necesidades. Ejemplos de estas especificaciones provienen del } \\
\text { Instituto Mexicano de la Construcción del Acero (Especificacio- } \\
\text { nes imca-2003) y especificaciones del Instituto Americano del } \\
\text { Hierro y el Acero (Especificaciones aisc, 2005). }\end{array}$ & Alto \\
\hline
\end{tabular}

Fuente: elaboración propia con base en los criterios de Gereffi et al (2005) y entrevistas

Revisando la cadena de valor del acero en México, con base en la documentación analizada y en las entrevistas es posible observar en los cuadros 1, 2 y 3 que los criterios de complejidad y codificación de información son altos, mientras que la capacidad de proveedores es baja. Particularmente, Gereffi (2005) menciona que cuando los criterios toman esos valores entonces se tiene una gobernanza del tipo cautiva Por lo tanto, es posible establecer que la gobernanza de la cadena de valor del acero de este país es del tipo cautiva, en la que la empresa líder busca impulsar el desarrollo de las pymes de su cadena mediante programas puntuales de transferencia de conocimiento, capacitaciones, asesoramiento, etcétera 


\section{Cuadro 2 \\ Criterios de gobernanza de la cadena de valor delaceroen México según el criterio 2}

\begin{tabular}{l|l|l}
\hline Criterio & Presencia en la cadena & Eva. \\
\hline $\begin{array}{l}\text { Codificación la } \\
\text { de la } \\
\text { información }\end{array}$ & $\begin{array}{l}\text { La codificación de información es alta y esto se puede ver en el } \\
\text { hecho de que la empresa líder tiene definidas especificaciones } \\
\text { puntuales para solicitar a sus proveedores y también las que tiene } \\
\text { que cumplir con sus clientes. Aunado a que estas especificaciones } \\
\text { son una guía importante para la transmisión de la información, } \\
\text { existe la complicación de que los procesos y productos se vuelven } \\
\text { tan especializados que pueden llegar a existir dificultades para que } \\
\text { los proveedores y clientes asimilen por completo las especifica- } \\
\text { ciones; entonces, para subsanar esta situación la empresa líder } \\
\text { ha generado programas especiales para trabajar con las pymes de } \\
\text { su cadena de valor. Particularmente estos programas involucran } \\
\text { soporte, seguimiento técnico, ayuda en optimización de procesos } \\
\text { y visitas a plantas industriales para transferir conocimiento tanto } \\
\text { en procesos como en productos. }\end{array}$ & \\
\hline
\end{tabular}

Fuente: elaboración propia con base en los criterios de Gereffi et al (2005) y entrevistas

\section{Cuadro 3}

\section{Criterios de gobernanza de la cadena de valor del acero en México según elcriterio 3}

\begin{tabular}{l|l|l}
\hline Criterio & Presencia en la cadena & Eva. \\
\hline Capacidad de & $\begin{array}{l}\text { La capacidad de los proveedores pymes es baja y esto se puede } \\
\text { proveedores }\end{array}$ & Bajo \\
ver en el hecho de que se han generado programas especiales para \\
que la empresa líder del acero ayudea las pymes en su cadena de \\
valor en su capacidad de proveeduría para cumplir con las especi- \\
ficaciones y normas correspondientes que cambian debido a que \\
las necesidades de los clientes son muy diversas y evolucionan. \\
Particularmente, estos programas para pymes involucran visitas a \\
plantas industriales para transferir conocimiento, capacitaciones \\
anuales hechas a la medida, asesoramiento y financiación en la \\
implementación o mejora de sistemas de gestión de calidad (cer- \\
tificación de normas de calidad iso 9001), seguridad (certificación \\
de normas de oshas 18001) y medio ambiente (certificación de \\
normas iso 14000).
\end{tabular} \mid

Fuente: elaboración propia con base en los criterios de Gereffi et al (2005) y entrevistas 


\section{Discusión}

Un análisis más profundo permite observar que el hecho de que la cadena de valor de los procesos productivos del acero tenga una gobernanza cautiva se da porque los proveedores pymes tienen una capacidad baja, ya que es difícil para ellas cumplir con las normas y especificaciones correspondientes para realizar actividades que ayuden a generar productos complejos como el acero, y esto presiona a la empresa líder a que intervenga en el desarrollo de los proveedores pymes Asimismo, el control por parte de la empresa líder fomenta el incremento de una dependencia de los proveedores y hace que sea muy costoso para los proveedores intentar cambiarse con otro cliente

La situación donde los costos de cambios son altos permite identificar a la empresa líder como el actor que tiene mayor poder, debido a que esta organización será la encargada de moldear la distribución de ganancias y riesgos (Gereffi, 2014). Particularmente, en el caso de la cadena de valor del aceroen México, laempresa líder es la que tiene mayor poder, yaque esta organización es quien toma la decisión respecto a qué proveedores apoyará mediante sus programas de desarrollo para que puedan obtener mayores ganancias

El usar la postura de Gereffi (2014) para establecer que la empresa líder en gobernanza cautiva tiene el mayor poder debido a los altos costos de cambio, está alineado a lo encontrado por Romero (2009), ya que él establece que en las gobernanzas de tipo cautiva son las empresas líderes las que asumen la organización de la división del trabajo entre las empresas participantes, ejerciendo un mayor o menor control sobre el desarrollo de las actividades y la estructura de la cadena Incluso Romero (2009) menciona que este papel protagonista le permite a la empresa líder retener una mayor porción del valor añadido total generado en la cadena Por lo tanto, se podría establecer que en la cadena de valor del acero la empresa líder tiene grandes beneficios al tener una gobernanza del tipo cautiva, debido a que esto le ayuda a generar mayor valor respecto al resto de los participantes, sobre todo que esta industria se basa en normas técnicas internacionales porque son productos altamente estandarizados

En mayor o menor grado las pymes que participan en la cadena de valor asumen generalmente un papel dependiente frente a la empresa líder que coordina con mayor poder en la gobernanza del tipo cautiva Esta relación 
de interdependencia asimétrica genera diferentes oportunidades y amenazas para las pymes que participan en la cadena

En cuanto a la oportunidad de ventaja que puede destacarse para las pymes que pertenecen a las cadenas de valor del tipo cautiva, está el favorecer sus posibilidades de crecimiento, debido a que la empresa líder buscaráimpulsar el desarrollo de proveedores locales para mantener su posición (Altenburg, 2006) Por lo tanto, se puede establecer que para el caso de la cadena de valor del acero las pymes salen beneficiadas por el hecho de que exista una gobernanza del tipo cautiva, ya que la empresa líder busca cooperar con ellas para impulsar su crecimiento y que se generen efectos directos sobre las ventas yel número de empleados Cabe señalar que esto es resultado de un análisis de la literatura y de la caracterización de la industria del acero; como una segunda etapa de investigación se podrá hacer un estudio correlacional con una población específica de las pymes de este sector para analizar su efecto inmediato.

El hecho de que la empresa líder del acero impulse a las pymes que forman parte de la cadena de valor es de gran ayuda, ya que los procesos de mejora en las pymes se han visto estimulados de la siguiente manera:

- La transferencia y asimilación tecnológica (efectos de difusión tecnológica) a partir de que la empresa líder ha generado programas de visitas de las pymes a plantas industriales para transferirconocimiento

- Los procesos de aprendizaje de los trabajadores de las pymes con capacitaciones hechas a la medida por parte de la empresa líder

- La ayuda de la empresa líderpara que las pymes obtengan estándares técnicos que provienen del Instituto Mexicano de la Construcción del acero

Previamente se han mencionado las ventajas y procesos de mejora que tienen las pymes que forman parte de la cadena de valor del acero con una gobernanza cautiva Sin embargo, Humphrey y Schmitz, (2000) establecen que esta forma de gobernanza también genera amenazas estratégicas para las pymes debido a que la capacidad de negociación de la que disfruta la gran empresa dificulta la retención de valor agregado por parte de las pymes.

Por último, se puede mencionar que la cadena de valor del acero tiene una gobernanza del tipo cautiva donde la empresa líder es la que tiene el mayor poder Asimismo, este tipo de gobernanza genera oportunidades para las pymes respecto al hechode que la empresalíder buscaráimpulsar el creci- 
miento y mejoras en los procesos de las empresas participantes en la cadena Sin embargo, el tener una gobernanza cautiva genera una desventaja para las pymes debido a que ellas tendrán una baja capacidad de negociación con la empresa líder

\section{Conclusiones}

La pregunta de investigación planteada en este estudioha sidorespondida debido a que se describióla cadena de valor del acero en México y fue posible identificarque lacadena tiene una gobernanza del tipo cautiva. Al estudiarla cadena del acero desde la perspectiva de su tipo de gobernanza fue posible percatarse de que los proveedores tienen una capacidad baja para realizar actividades que ayuden a generar productos complejos como el acero, y esto presiona a la empresa líder a que intervenga en el desarrollo de los proveedores

El objetivo del estudio fue alcanzado debido a que se identificó que la cadena de valor del acero en México tiene una gobernanza del tipo cautiva y se encontró que esta gobernanza tiene la particularidad de que genera ventajas para las pymes, debido a que la empresa líder buscaráimpulsar el crecimiento y mejoras en los procesos de sus proveedores

Finalmente, se puede establecer que la limitación de esta investigación es que está basada en un caso de estudio acotado al acero, pero sin ver el impacto que tienen las políticas públicas para incentivar que las empresas líderes ayuden a desarrollar a las pymes que participan en la cadena Incluso se considera que una futura línea de investigación seria identificar todos los incentivos generados por la política pública para ayudar al crecimiento y mejora de los procesos de los participantes de la cadena de valor, pero dándole seguimiento a la forma en la que estos incentivos empoderan a las pymes para incrementar su capacidad de negociación respecto a la empresa líder

\section{Referencias bibliográficas}

Altenburg, T (2006) "Governance Patterns in Value Chains and their Development Impact", European Journal of Development Research, 18(4): 498-521

Altos Hornos de México (2013) Manual de diseño para la construcción con acero Extraido (febrero 2015) de http://www ahmsa com/Acero/Complem /Manual_Construccion_2013/manual_ahmsa_2013 pdf 
Baxter, P, y Jack, S (2008) "Qualitative case study methodology: Study design and implementation for novice researchers", The Qualitative Report, 13(4): 544-559

Camacho, L. F.(2011). "Reflexión sobre la industria del acero en el mercado globalizado", Apuntes del cenes, 30(51): 165-182

Canacero (2011) www.canacero.org. Recuperado el 21 de abril de 2015, de www canacero org: y http://www mejorconacero com/wp-content/uploads

_ (2013) Perfil de la industria siderúrgica en México (2004-2013) (2015) www.canacero.org.mx Recuperado el 20 de abril de 2015, de www canacero org mx: http://www canacero org mx/Es/sustentabilidad html

Chiviri, E , y García, D (2011) “Industria siderúrgica y mercado del acero: Caracterización y perspectivas”, Informe Económico, núm 20, pp 1-9

Gereffi, G. (2005). "The global economy: Organization, governance and development”, en Smelser, N, y Swedberg, R (Eds), Handbook of Economic Sociology Princeton: Princeton University Press, pp 160-182

(2014) "Global value chains in a post-Washington Consensus world", Review of International Political Economy, núm 21, pp9-37

Gereffi, G., Humphrey, J., y Sturgeon, T. (2005). "The governance of global value chains", Review of International Political Economy, 12(1): 78-104

Humphrey, J , y Schmitz, H (2002) "How does Insertion in Global Value Chains Affect Upgrading Industrial Clusters?”, Regional Studies, 36(9): 1017-1027

Industrial, I (2014) El ciclo de vida del acero y su impacto en la cadena de valor Extraído de: http://www informeindustrial com ar/verNota aspx?nota=El ciclo de vida del acero y su impacto en la cadena de valor 579

Iris. (2013) Informe 2013 sobreel reciclaje del aceroen laindustria siderúrgica Recuperado el 29 de mayo de 2015, de http://www unesid org/iris2013/elacero html

Kaplinsky, R, y Morris, M (2009) Un manual para investigación de cadenas de valor

Romero, I (2009) "Pymes y cadenas de valor globales: Implicaciones para la política industrial en las economías en desarrollo", Análisis Economico, 24(57): 199-216

Yin, R K (2003) Case study research: Design and methods, $3^{\mathrm{a}}$ edición Thousand Oaks ca: Sage 